\title{
The "Sleep Well, Lincolnshire" Project: Evaluation of an Online Sleep Practitioner Clinic
}

\author{
Heather Elphick ${ }^{1}$, Claire Earley ${ }^{2}$, Karen Tyas ${ }^{2}$, Lowri Thomas ${ }^{1}$, Lisa Artis ${ }^{2}$, Vicki Dawson ${ }^{2}$ \\ ${ }^{1}$ Department of Sleep Medicine, Sheffield Children's NHS Foundation Trust, Sheffield, \\ ${ }^{2}$ The Sleep Charity, Doncaster, UK
}

Received June 11, 2021

Revised August 15, 2021

Accepted September 9, 2021

Address for correspondence

Heather Elphick,

$\mathrm{MD}, \mathrm{MB}, \mathrm{ChB}, \mathrm{MRCP}$

Department of Sleep Medicine,

Sheffield Children's NHS

Foundation Trust,

Western Bank, Sheffield,

S10 2TH, UK

Tel: +44 1142717400

E-mail: h.elphick@nhs.net
Objectives: Poor sleep is associated with adverse outcomes during childhood. Behavioral insomnia is the most common sleep difficulty experienced by children. The coronavirus disease 2019 (COVID-19) global pandemic in 2020 has profoundly affected children's sleep patterns. This project aimed to evaluate a one-toone sleep service delivered via online clinics by community sleep practitioners in the UK. Methods: This was an observational pre- and post-evaluation study over a 12-month period. The intervention derived from aspects of cognitive-behavioral therapy for insomnia. The evaluation was questionnaire-based and assessed sleep parameters and well-being. Results: 104 parents returned completed questionnaires. The average time of sleep onset was 1 hour and 39 minutes pre-intervention and 20 minutes post-intervention. The average number of nights per week that children woke up was 3.9 pre-intervention and 0.9 post-intervention; the number of night awakenings fell from 1.9 to 0.5 and the time that children were awake after sleep onset fell from 66.8 minutes to 5.8 minutes. The average time that children were asleep was 8.0 hours per night pre-intervention and 10.2 hours post-intervention. The improvement in all sleep parameters was statistically significant $(p<0.05)$. All parameters of parental and children's well-being improved significantly $(p<0.05)$, except for perceived ability to drive $(p=0.07)$. All parents stated that they would recommend sleep support and 20\% already had done so. Conclusions: The COVID-19 pandemic has accelerated the development of remote health care solutions, and in the case of children's sleep clinics, the online mode of intervention delivery that is as effective, acceptable, and accessible as face-to-face delivery.

J Sleep Med 2021;18(3):154-161

Keywords: Sleep; Children; Delivery of health care; COVID-19; Mental health.

\section{INTRODUCTION}

Sleep is a restorative process that is fundamental to physical and psychological health. ${ }^{1}$ The most common sleep difficulties experienced by children are bedtime resistance and nighttime awakening, known as behavioral insomnia. The prevalence of insomnia in children is reported to be in the region of $30 \%,{ }^{2,3}$ and up to $86 \%$ in children with neurodevelopmental disorders, including autism and attention-deficit/hyperactivity disorder (ADHD). ${ }^{4,5}$

Poor sleep is associated with several adverse short- and longterm outcomes in childhood, including problems with behavior, emotion regulation, and school performance, ${ }^{6,7}$ as well as an increased risk of accidents or injuries, poorer physical health,

This is an Open Access article distributed under the terms of the Creative Commons Attribution Non-Commercial License (https://creativecommons.org/licenses/by-nc/4.0) which permits unrestricted non-commercial use, distribution, and reproduction in any medium, provided the original work is properly cited. and poorer mental health outcomes. ${ }^{8,9}$ Sleep disruption in children affects their parents' mood and emotion regulation..$^{10} \mathrm{An}$ ecdotally, these types of stress lead to an increased demand for NHS services and prescriptions of drugs such as antidepressants.

Children's sleep difficulties often arise from the way that parents manage their children's sleep ${ }^{11}$ and their response to behavioral therapy. ${ }^{12}$ Sleep practitioner-led community sleep programs are highly effective at improving sleep onset times, sleep duration, and the well-being of children and parents. ${ }^{13,14}$

The coronavirus disease 2019 (COVID-19) became a global pandemic in 2020, and to reduce the spread of the virus, people were required to stay at home. This resulted in school and nursery closures that affected the well-being of many children. Emerging evidence from recent research and surveys shows that the COVID-19 pandemic has had a profound effect on all aspects of social life, including mental and physical health. ${ }^{15}$ An online survey that explored the changes in sleep patterns 
in children in the UK during the COVID-19 pandemic showed that $20 \%$ of children shifted their sleep onset by more than two hours later, regular bedtimes reduced from over $90 \%$ to $50 \%$, and nearly half of the children were having less sleep time overall than before the pandemic. The incidence of nightmares, night-time awakenings, and children asking their parents to be with them at bedtime or during the night increased (unpublished work). In several cases, sleep difficulties have been linked to increased anxiety, both as a cause and a consequence. This creates a vicious cycle that could have profound effects on the mental health of children.

With the advent of COVID-19, it has become imperative for health care services to rapidly embrace digital solutions. Faceto-face community sleep support clinics have not been possible during the pandemic, and methods of online learning and service delivery have emerged as a matter of urgency and necessity. This project aimed to evaluate a one-to-one sleep service delivered via online clinics by community sleep practitioners in the UK.

\section{METHODS}

This study was designed as an observational pre- and postevaluation of a behavioral sleep intervention that occurred in the form of an online video-clinic appointment using the Microsoft Teams platform with follow-up phone support. Practitioners were present throughout each consultation session for "live" video chat; no session was pre-recorded. No control group was included. NHS research ethics were consulted and approvals were not required as the participants were recruited and treated through a charity and were not NHS patients. Information about the study and general data protection regulation was provided, and written consent was obtained.

\section{Recruitment}

A consecutive cohort of children and young people under 16 years old with sleep problems was recruited via self-referral to the "Sleep Well Lincolnshire" project. The project was advertised via social media, tagging in local groups such as healthcare organizations and charities, press releases in local papers and radio stations, posters and flyers displayed across the area, and on The Sleep Charity website. Children with a specific physiological sleep or medical disorder (e.g., sleep apnea, pain, respiratory or gastrointestinal symptoms, or seizures) were excluded if sleep disturbance was thought to have a medical basis that should be prioritized over the sleep support intervention, as screened for by the practitioners at the initial assessment and discussed with the consultant (HE). Children were referred to the child's general practitioner $(\mathrm{n}=2)$, child and adolescent mental health services $(n=1)$, or the sleep clinic at Shef- field Children's Hospital as necessary $(n=2)$. Children with other sleep problems, such as sleepwalking, were included if it was considered that they may still benefit from the sleep support intervention with no other concerns identified. Children with any other comorbidities, including neurodevelopmental conditions, were included.

\section{Intervention}

Three experienced sleep practitioners delivered sleep support interventions over a 12-month period. The practitioners had previously undergone accredited sleep practitioner training through The Sleep Charity (www.thesleepcharity.org.uk). The objective of the intervention was to provide bespoke support from practitioners to parents/caregivers and/or young people, with the aim to train and empower them to implement a behavioral sleep program at home to improve their children's night-time behavior. Delivery was targeted at parents, in the case of children 0-11 years old, with children either present or absent, depending on parental preference, and at young persons, 12-16 years old with one parent present. Teenagers were encouraged to develop their own sleep program with the practitioner supporting them to devise their own solutions.

The intervention was a supportive non-judgmental approach using a one-to-one clinical model. Key elements of the intervention were education about basic sleep physiology, a detailed assessment, and the development of an individualized sleep plan, which was communicated either by email or post. If available, the practitioner observed the children's behavior by interacting with them and watching them play, and assessed the bedroom environment through an online camera in order to provide bespoke advice for each child. The plan included practical advice such as removal of technology-related devices and other stimulating objects from the bedroom, encouraging nonstimulating activities for 1 hour before bedtime, preparation of melatonin-producing dinner meals, and avoidance of caffeine.

Consultation was followed up with weekly phone calls and/ or emails to provide motivation, support, and further advice for six weeks. The intervention involved up to six sessions of up to 1 hour each. The end of the intervention was defined as the end of consultation number 6 , unless the parent/young person and practitioner agreed that no further sessions were necessary prior to this point.

\section{Evaluation}

Evaluations occurred before the intervention (pre-intervention), at six weeks after the start of the intervention (post-intervention), at the end of the intervention. Demographic information such as age, sex, medical diagnoses, and medications was collected at baseline.

Self-designed questionnaires were communicated to par- 
ents via email or post to examine:

- The child's sleep pattern, based on parental recollection, including time required to settle down, time required to fall asleep, number of nights per week that the child woke up during the night, number of awakenings per night, duration of nocturnal awakenings, and total sleep duration per night.

- Parent/caregiver well-being - a rating of their quality of life in the previous two weeks, based on a series of factors rated between 1-5, with the lower number indicating better well-being.

- The child's well-being - a rating of their quality of life in the previous two weeks, based on a series of factors rated between $1-5$, with the lower number indicating better well-being.

- Improvement in parents' understanding of their child's sleep problems and their confidence in the management of their children's sleep during the follow-up.

Feedback on the intervention (including negative feedback) was also assessed during the follow-up evaluation.

\section{Analysis}

The number of families that were recruited depended on the number of clinics that were able to provide support during the initial study period of 12 months.

Only completed evaluations before and after the sleep support intervention were included. Missing data were taken into account by imputing the baseline value into the final outcome data (or vice versa) for the sleep measures. Average score values were imputed for the well-being questionnaires. A per-protocol analysis of the patients who completed the intervention and evaluation was performed. For comparison of scores from baseline to post-intervention, the mean differences, standard deviations, and 95\% confidence intervals were calculated using Excel. Skewness and kurtosis were calculated using Excel for each variable and were found to be $>2$ in most cases, indicating that the data were not normally distributed. The nonparametric Wilcoxon signed-rank test was, therefore, used for statistical analysis (Social Science Statistics, https://www.socscistatistics.com/). Mean differences in the sleep data and total well-being scores for parents and children were compared for all age groups using the Mann-Whitney $\mathrm{U}$ test. The significance threshold was set at $p<0.05$.

\section{RESULTS}

In total, 260 parents self-referred to The Sleep Charity's Lincolnshire Sleep Clinic between February 2020 and February 2021. Fig. 1 shows the number of families that did not complete the evaluation for any reason.

A total of 104 parents returned completed forms before and at the end of the sleep intervention. The average age of children was 12 years (range, 2 months-16 years) with 87 children being under 11 years old and 17 young people 12-16 years old. A total of 55 children were normally developing with no reported underlying diagnosis; 44 had a neurodevelopmental or medical diagnosis, including autism $(n=16)$, anxiety $(n=2)$, $\operatorname{ADHD}(n=10)$, autism and $\operatorname{ADHD}(n=4)$, unspecified mental health illness $(n=2)$, Down syndrome $(n=1)$, and others $(n=9)$. Five children were assessed for possible neurodevelopmental diagnoses. Thirteen children were taking melatonin, 87 children had no medication for their sleep, one was taking chloral hydrate, and three children were prescribed melatonin but

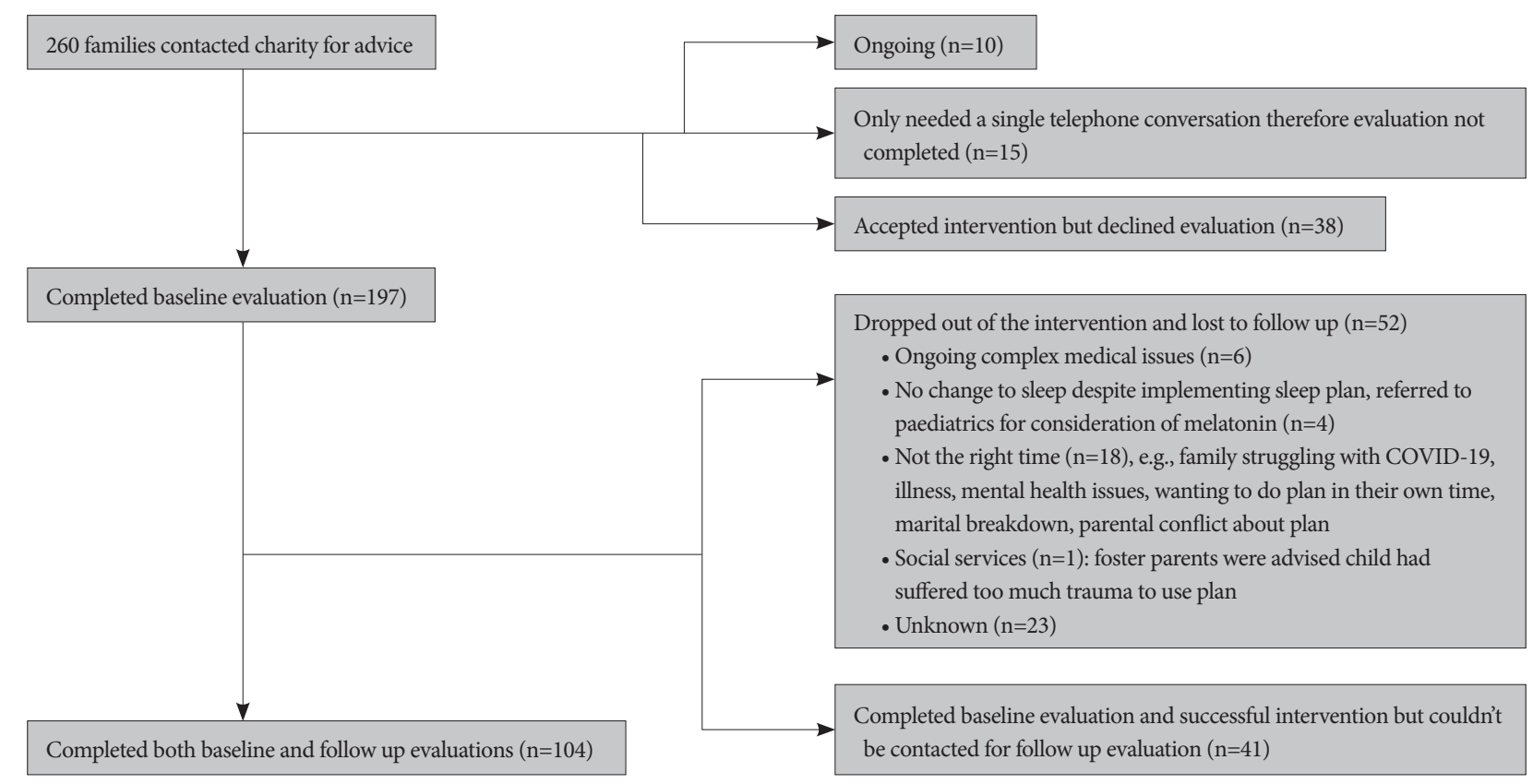

Figure 1. Flowchart to demonstrate the number of families that did not complete the evaluation with accompanying reasons. 
not taking it.

\section{Sleep data}

Table 1 shows the sleep measures before and at the end of the sleep intervention. The average time required for children to fall asleep on a typical night was 99 minutes (1 hour and 39 minutes) before the sleep support intervention. At the end of the intervention, the average time required for children to fall asleep was 20 minutes. This was an improvement by 1 hour and 19 minutes.

The average number of nights per week that children woke up was 3.9 before the intervention and 0.9 after the intervention; the number of awakenings per night fell from 1.9 to 0.5 and the time that children were awake during a typical night fell from 66.8 minutes to 5.8 minutes.

The average time that children were asleep per night was reported as 8.0 hours before the sleep intervention and 10.2 hours at the end of the intervention. This was an average increase of 2.2 hours of sleep per night.

All parameters of sleep improved significantly after the sleep intervention $(p<0.05)$. The results were not significantly different between children $0-11$ years old and children 12-16 years old.

\section{Parental well-being}

Table 2 shows parental well-being parameters before and at the end of the sleep intervention. All parameters of parental well-being improved significantly following the sleep intervention $(p<0.05)$, except for their perceived ability to drive $(p=0.07)$. The total well-being score was not significantly different between children age groups $(p=0.34)$.

The greatest improvement was observed (highest mean difference) in parental reported stress.

\section{Children's well-being}

Table 3 shows children's well-being parameters before and at the end of the sleep intervention All parameters of children's well-being improved significantly following the sleep intervention $(p<0.05)$. The total well-being score was not significantly different $(p=0.25)$ between children $0-11$ years old and 12-16 years old.

The greatest improvement was observed (highest mean difference) in reported daytime behavior and happiness.

\section{Parents' understanding and confidence}

At the end of the sleep support, parents rated their improvement in their understanding of their child's problems as 9.6/10 and improvement in their confidence to deal with children's problems as 9.6/10. All parents stated that they would recommend sleep support, and $20 \%$ already had done so.

Parents commented on what was most helpful and what could have been improved. The five most common themes for

Table 1. Sleep measures before and after the sleep intervention $(n=104)$

\begin{tabular}{lccc}
\hline \multicolumn{1}{c}{ Variable } & Baseline (mean) & Post-intervention (mean) & Difference (mean \pm SD) \\
\hline Time to fall asleep (min) & 99.26 & 20.38 & $78.88 \pm 69.81^{*}$ \\
Number of nights wake per week & 3.92 & 0.85 & $3.08 \pm 2.95^{*}$ \\
Number of awakenings per night & 1.88 & 0.45 & $1.42 \pm 2.39^{*}$ \\
Time awake during night (min) & 66.78 & 5.75 & $60.39 \pm 93.82^{*}$ \\
Number of hours sleep (hr) & 7.98 & 10.18 & $2.20 \pm 1.96^{*}$ \\
\hline
\end{tabular}

$* p<0.05$

Table 2. Parental well-being measures before and after the sleep intervention $(n=92)$

\begin{tabular}{lccc}
\hline \multicolumn{1}{c}{ Variable } & Baseline (mean) & Post-intervention (mean) & Difference (mean \pm SD) \\
\hline Makes me feel isolated & 3.04 & 1.51 & $1.47 \pm 1.32^{*}$ \\
Makes me feel stressed & 3.92 & 1.72 & $2.11 \pm 1.08^{*}$ \\
Affecting self-esteem and confidence & 2.91 & 1.55 & $1.30 \pm 1.27^{*}$ \\
Affecting ability to cope with everyday life & 3.22 & 1.59 & $1.56 \pm 1.25^{*}$ \\
Impacting on relationship with child & 3.05 & 1.50 & $1.49 \pm 1.32^{*}$ \\
Impacting on relationship with family & 2.76 & 1.58 & $1.14 \pm 1.27^{*}$ \\
Impacting on relationship with partner & 3.20 & 1.69 & $1.46 \pm 1.30^{*}$ \\
Impacting on ability to drive safely & 1.85 & 1.60 & $0.24 \pm 1.02(p=0.07)$ \\
Affecting my health & 2.66 & 1.53 & $1.08 \pm 1.22^{*}$ \\
Total score & 26.62 & 14.22 & $11.89 \pm 6.86^{*}$
\end{tabular}

Each question was rated on a scale of $1-5$ where 1 was good and 5 was poor. ${ }^{*} p<0.05$ 
Table 3. Children's well-being measures before and after the sleep intervention $(n=90)$

\begin{tabular}{lccc}
\hline \multicolumn{1}{c}{ Variable } & Baseline (mean) & Post-intervention (mean) & Difference (mean \pm SD) \\
\hline Affecting self-esteem \& confidence & 2.81 & 1.69 & $1.06 \pm 1.41^{*}$ \\
Affecting daytime behaviour & 3.86 & 1.83 & $1.92 \pm 1.11^{*}$ \\
Impacting on health & 2.40 & 1.53 & $0.82 \pm 1.25^{*}$ \\
Impacting on happiness & 3.39 & 1.53 & $1.76 \pm 1.24^{*}$ \\
Impacting on ability to concentrate & 3.01 & 1.86 & $1.09 \pm 1.35^{*}$ \\
Impacting on relationship with family & 3.29 & 1.69 & $1.52 \pm 1.20^{*}$ \\
Total score & 17.05 & 9.21 & $8.17 \pm 5.31^{*}$ \\
\hline
\end{tabular}

Each question was rated on a scale of $1-5$ where 1 was good and 5 was poor. ${ }^{*} p<0.05$

what was most helpful were: 1) the detailed and individualized sleep plan, 2) the follow-up support, 3) being able to talk and be treated with empathy and no judgement, 4) understanding the science and having a "light-bulb moment," and 5) practical advice and tips, such as the sleep box, screen-free time, and sleep-promoting meals.

Only one of the 104 parents thought that more help was required for her child, who was still waking up during the night. There were only three other comments relating to potential improvements, including additional funding for the program and availability to more people.

\section{Feedback}

Free-text comments included comments referring to improvements in the well-being of children, parents, siblings, and the family as a whole (Supplementary Table 1 in the onlineonly Data Supplement). There were also improvements regarding mental and physical health, school attendance and achievements, and specific comments about foster placement and the impact on parents' driving.

\section{DISCUSSION}

To our knowledge, this is the first report of a video-based clinic developed for behavioral insomnia in children during the COVID-19 pandemic in the UK. The key findings from this evaluation were that, with an online individualized sleep program and support from a sleep practitioner delivered via a video platform and phone calls, children's sleep and well-being and parent/caregiver well-being were significantly improved. In addition, parents understood better their child's sleep problem, became more confident in managing their children's sleep problems after the intervention, and would recommend the service to other parents.

The finding that a behavioral intervention for sleep support is effective is not novel. Meltzer et al. ${ }^{12}$ reviewed 120 studies in 2020 and found considerable heterogeneity in terms of patient characteristics, treatment approaches, and outcome measures, highlighting the complexity of the intervention approach. Gaps in the research were identified and recommendations were made for future research, such as the inclusion of measures regarding the mood, behavior, and quality of life, and alternative treatment delivery modes with the aim to increase access to care for children and their families. ${ }^{12}$

One such alternative delivery mode is remote health care for insomnia in children. Remote health care approaches offer several potential additional advantages over conventional faceto-face methods, including convenience and improved access to families, lower cost, and travel savings, making them more environmentally friendly and time-efficient. ${ }^{16}$ Other potential benefits include reduced absence from school and parents' work, ability to bring together caregivers and the professional multidisciplinary team when needed; in addition, the widespread use of smartphones among caregivers promotes engagement, leading to reduced non-attendance rates. Consultations take place with children at home, where they can be more relaxed. Moreover, practitioners can observe children while they are playing and may have a view of their bedroom, if needed. This approach was introduced in our study, but it should be explored in more detail in future research. Potential disadvantages include internet speed problems, parent and child acceptability of the video platform, equipment malfunction, and the cost of installing the equipment.

Telemedicine has previously been used to evaluate and treat insomnia in children, but the relative effectiveness of different approaches has not yet been studied. ${ }^{17}$ Treatment methods using audio recordings have led to improvements in subjective sleep diaries and questionnaire measures, ${ }^{18}$ and a six-week webbased program has also led to improved objective sleep measures. ${ }^{19}$ The use of more interactive methods, such as cognitive behavioural therapy for insomnia for children and adolescents $^{20}$ using videos, automated and written feedback, interactive questionnaires, and individualized advice showed, in a randomized controlled trial, that the children who received this advice had significantly improved sleep compared to the waiting-list control group, although total sleep time did not 
change. Three studies on direct remote health care also showed improvements in sleep outcomes ${ }^{21-23}$ as well as improved access to families. ${ }^{24}$ An online teaching program that was identical to the face-to-face program led to improvement in sleep scores, similar to the face-to-face group and decreased the number of overnight awakenings. ${ }^{21}$ The Pediatric TeleSleep program used direct video conferencing technology to address sleep problems in children in Canada for families unable to access healthcare due to distance, and demonstrated improved access for families located in rural settings. ${ }^{24}$ The Better Nights/Better Days project analyzed five weekly telephone coaching sessions delivered by trained sleep coaches and found an improvement in sleep measures compared with the waiting-list control group with participants reporting the intervention to be usable, useful, acceptable, and feasible. ${ }^{22,23}$ The use of a combination of a video platform for the initial assessment and phone call follow-up for delivery of support in our study has shown similar success in terms of sleep and well-being outcome measures and has confirmed that the video platform was accessible to parents with the additional benefit that the sleep practitioner was able to watch children and their environment during the assessment.

Although rarely employed prior to the pandemic due to perceived difficulties with acceptance and logistics, remote health care solutions are now important, because clinics were closed and the public was told to stay at home as a result of the COVID-19 pandemic. ${ }^{25}$ In response to the pandemic, during which families have been unable to access face-to-face support, the Mini-KiSS Online project group adapted their 2012 program and performed a pilot evaluation with 12 parents and 6 children with significant sleep problems. Sleep training was accomplished with online group sessions with 2-3 parents per group, and videos were prepared for the children. Early evaluation results were very positive in terms of both the acceptability of the online nature of the course and the improvement in sleep. ${ }^{26}$

Our study addressed the response needed due to the COVID-19 pandemic in a real-world situation with a face-to-face sleep clinic being rapidly converted to a video clinic. The study attempted to follow some of Meltzer's recommendations ${ }^{12}$ in that it explored the use of an alternative delivery platform and assessed the improvement in non-sleep as well as sleep parameters. This is the first report of an e-consultation and tele-education sleep clinic with telephone support for families in the UK during the COVID-19 pandemic, which, although not a directly controlled trial, has shown similar results to those from a previous evaluation of a face-to-face clinic model using the same methodology. ${ }^{13}$ This study has shown that the delivery and implementation of sleep support using an online model is feasible for children in the community. The support was delivered by The Sleep Charity's experienced and skilled sleep practitioners from a variety of non-health backgrounds who have undergone rigorous accredited training, including training in recognition of medical, psychological, and safeguarding concerns with appropriate signposting.

Two hundred and sixty families self-referred to the clinic in this study, and of these, 197 provided consent to the full intervention and evaluation. Only $53 \%$ of the recruited participants completed the full intervention and evaluation. Fifty-two parents failed to complete the intervention. The majority of these stated that it was not a good time for them to implement the intervention for a range of health and social reasons; other reasons for failure to complete the intervention were associated with medical complexity, with a small proportion of families seeking further medical advice, and consideration for melatonin prescription. The remaining 41 parents completed the intervention with a successful outcome as assessed by the sleep practitioner but did not complete the follow-up evaluation. Disengagement in this subgroup was, therefore, more likely to be associated with the research process than the sleep intervention itself. Due to the withdrawal rate, a per-protocol analysis was performed on participants who completed the intervention and provided data on the outcome measures. However, this type of analysis represents the maximum potential or "best case" results. In addition, imputed average scores may have artificially inflated pre-intervention scores and reduced post-intervention scores. Attrition rates were also high in previous studies; for example, Roberts et al. ${ }^{21}$ showed that only $67 \%$ of parents completed all training sessions, and in our previous work, ${ }^{13}$ in which the intervention was delivered face-to-face, there was a 30\% withdrawal rate from the intervention. We conclude from this that the reasons for failure to complete this type of intervention are likely to be unrelated to the mode of delivery of the support, but more likely related to the research processes, such as the administration of questionnaires via email or post.

Caution is needed when interpreting the results of this observational study. Because of the rapid implementation of this service, the study was not designed as a randomized controlled evaluation, but in a real-world setting; however, we think it is valid and appropriate to report our results, to highlight the effectiveness of this mode of delivery and to promote future trials. No parents declined the intervention due to its online format, and challenges such as loss of internet connection did not impair the progress of the project. Without a concurrent control group, it cannot be assumed that the observed changes were directly due to the intervention. While the results may have been influenced by other interventions introduced concurrently at the time of the study, it is likely, and as verified by the parents' reports, that it was indeed the sleep intervention that led to significant sleep improvement in these children. An additional limitation of our study was subjective parental reporting with no objective measures. We overcame this limita- 
tion by asking the same parent to assess their child before and after the intervention, thus ensuring consistency in the responses in the absence of an objective measure. Some questions, such as "affecting self-esteem and confidence," may not have been appropriate across all ages, e.g., for children under two years old, although, interestingly, 11/12 parents responded to this question. In our experience, requests to complete a battery of questionnaires prior to initial consultation have proved to be a barrier for families in this type of community, leading to high attrition rate; therefore, for this evaluation, the length of the questionnaires was kept to a minimum.

The overall aim of our service is to provide early intervention sleep support that is accessible to all children, young people, and their families across the county to help avoid long-term serious sleep issues. We also aim to provide data to encourage local commissioners to fund sleep support in the future to ensure sustainability. The COVID-19 pandemic has accelerated the development of remote health care solutions and, in the case of children's sleep clinics, has provided a mode of intervention delivery that appears to be as effective, acceptable, and accessible as face-to-face delivery. We aim to integrate the online sleep clinic into the existing workforce with peer mentoring and volunteering, which will ensure sustainability. Locally, we will share the information with the commissioners and head of services across the region, and we hope that other regions will be keen to adopt our approach. The long-term efficacy of this treatment modality will require robustly designed trials, and an in-depth, economic analysis is required to determine the delivery costs of this type of service and demonstrate potential cost-effectiveness.

\section{Supplementary Materials}

The online-only Data Supplement is available with this article at https:// doi.org/10.13078/jsm.210013.

\section{Conflicts of Interest}

The authors have no potential conflicts of interest to disclose.

\section{ORCID iDs}

Heather Elphick

Claire Earley

Karen Tyas

Lowri Thomas

Lisa Artis

Vicki Dawson https://orcid.org/0000-0001-8982-9250

https://orcid.org/0000-0001-7609-3117 https://orcid.org/0000-0003-3984-6655 https://orcid.org/0000-0001-6316-4684 https://orcid.org/0000-0002-4683-9659 https://orcid.org/0000-0003-1396-7280

\section{Author Contributions}

Conceptualization: Heather Elphick, Vicki Dawson, Lisa Artis. Data curation: Claire Earley, Karen Tyas, Lowri Thomas. Formal analysis: Heather Elphick. Funding acquisition: Vicki Dawson, Lisa Artis, Heather Elphick. Project administration: Lowri Thomas. Supervision: Heather Elphick, Vicki Dawson. Writing_original draft: Heather Elphick. Writing_review \& editing: Vicki Dawson, Lisa Artis, Claire Earley, Lowri Thomas, Karen Tyas.

\section{Funding Statement}

This study was funded by The National Lottery Community Fund (grant number: 10371255).

\section{REFERENCES}

1. Gregory AM, Sadeh A. Annual research review: sleep problems in childhood psychiatric disorders--a review of the latest science. J Child Psychol Psychiatry 2016;57:296-317. https://doi.org/10.1111/jcpp.12469.

2. Dohnt H, Gradisar M, Short MA. Insomnia and its symptoms in adolescents: comparing DSM-IV and ICSD-II diagnostic criteria. J Clin Sleep Med 2012;8:295-299. https://doi.org/10.5664/jcsm.1918.

3. Williamson AA, Mindell JA, Hiscock H, Quach J. Child sleep behaviors and sleep problems from infancy to school-age. Sleep Med 2019; 63:5-8. https://doi.org/10.1016/j.sleep.2019.05.003.

4. Singh K, Zimmerman AW. Sleep in autism spectrum disorder and attention deficit hyperactivity disorder. Semin Pediatr Neurol 2015;22: 113-125. https://doi.org/10.1016/j.spen.2015.03.006.

5. Corkum P, Davidson FD, Tan-MacNeill K, Weiss SK. Sleep in children with neurodevelopmental disorders: a focus on insomnia in children with ADHD and ASD. Sleep Med Clin 2014;9:149-168. https://doi. org/10.1016/j.jsmc.2014.02.006.

6. Curcio G, Ferrara M, De Gennaro L. Sleep loss, learning capacity and academic performance. Sleep Med Rev 2006;10:323-337. https://doi. org/10.1016/j.smrv.2005.11.001.

7. Gaultney JF. The prevalence of sleep disorders in college students: impact on academic performance. J Am Coll Health 2010;59:91-97. https:// doi.org/10.1080/07448481.2010.483708.

8. Beebe DW. Cognitive, behavioral, and functional consequences of inadequate sleep in children and adolescents. Pediatr Clin North Am 2011;58:649-665. https://doi.org/10.1016/j.pcl.2011.03.002.

9. Owens J. Insufficient sleep in adolescents and young adults: an update on causes and consequences. Pediatrics 2014;134:e921-e932. https:// doi.org/10.1542/peds.2014-1696.

10. Byars KC, Yeomans-Maldonado G, Noll JG. Parental functioning and pediatric sleep disturbance: an examination of factors associated with parenting stress in children clinically referred for evaluation of insomnia. Sleep Med 2011;12:898-905. https://doi.org/10.1016/j.sleep.2011.05.002.

11. Meltzer LJ. Clinical management of behavioral insomnia of childhood: treatment of bedtime problems and night wakings in young children. Behav Sleep Med 2010;8:172-189. https://doi.org/10.1080/15402002.20 10.487464 .

12. Meltzer LJ, Wainer A, Engstrom E, Pepa L, Mindell JA. Seeing the whole elephant: a scoping review of behavioral treatments for pediatric insomnia. Sleep Med Rev 2021;56:101410. https://doi.org/10.1016/ j.smrv.2020.101410.

13. Elphick HE, Lawson C, Ives A, et al. Pilot study of an integrated model of sleep support for children: a before and after evaluation. BMJ Paediatr Open 2019;3:e00551. https://doi.org/10.1136/bmjpo-2019-000551.

14. Turner K, Bullock L, Down A, Brown K, Foulkes K, Thomson L. Sleep tight: an evaluation of a community based intervention on the sleep behaviours of young children. J Health Visit 2016;4:572-578. https:// doi.org/10.12968/johv.2016.4.11.572.

15. Holmes EA, O'Connor RC, Perry VH, et al. Multidisciplinary research priorities for the COVID-19 pandemic: a call for action for mental health science. Lancet Psychiatry 2020;7:547-560. https://doi.org/10.1016/ S2215-0366(20)30168-1.

16. Smith AC, Scuffham P, Wootton R. The costs and potential savings of a novel telepaediatric service in Queensland. BMC Health Serv Res 2007;7:35. https://doi.org/10.1186/1472-6963-7-35.

17. McLay L, Sutherland D, Machalicek W, Sigafoos J. Systematic review of telehealth interventions for the treatment of sleep problems in children and adolescents. J Behav Educ 2020;29:222-245. https://doi. org/10.1007/s10864-020-09364-8. 
18. Schlarb AA, Brandhorst I. Mini-KiSS Online: an internet-based intervention program for parents of young children with sleep problems influence on parental behavior and children's sleep. Nat Sci Sleep 2012; 4:41-52. https://doi.org/10.2147/NSS.S28337.

19. Mullane J, Corkum P. Case series: evaluation of a behavioral sleep intervention for three children with attention-deficit/hyperactivity disorder and dyssomnia. J Atten Disord 2006;10:217-227. https://doi. org/10.1177/1087054706288107.

20. de Bruin EJ, Bögels SM, Oort FJ, Meijer AM. Efficacy of cognitive behavioral therapy for insomnia in adolescents: a randomized controlled trial with internet therapy, group therapy and a waiting list condition. Sleep 2015;38:1913-1926. https://doi.org/10.5665/sleep.5240.

21. Roberts CA, Smith KC, Sherman AK. Comparison of online and faceto-face parent education for children with autism and sleep problems. J Autism Dev Disord 2019;49:1410-1422. https://doi.org/10.1007/s10803018-3832-2.

22. Corkum P, Lingley-Pottie P, Davidson F, et al. Better nights/better days-distance intervention for insomnia in school-aged children with/ without ADHD: a randomized controlled trial. J Pediatr Psychol 2016; 41:701-713. https://doi.org/10.1093/jpepsy/jsw031.

23. Tan-MacNeill KM, Smith IM, Weiss SK, et al. An eHealth insomnia intervention for children with neurodevelopmental disorders: results of a usability study. Res Dev Disabil 2020;98:103573. https://doi.org/10.1016/ j.ridd.2020.103573.

24. Witmans MB, Dick B, Good J, et al. Delivery of pediatric sleep services via telehealth: the Alberta experience and lessons learned. Behav Sleep Med 2008;6:207-219. https://doi.org/10.1080/15402000802371312.

25. Paruthi S. Telemedicine in pediatric sleep. Sleep Med Clin 2020;15:e1e7. https://doi.org/10.1016/j.jsmc.2020.07.003.

26. Schlarb AA, Schulte H, Selbmann A, Och I. Online cognitive behavioral group therapy (iCBT-I) for insomnia for school children and their parents: adaptation of an established treatment (KiSS training). Somnologie (Berl) 2020;24:259-266. https://doi.org/10.1007/s11818-020-00280-7. 
Supplementary Table 1. Free-text feedback from parents after the sleep intervention

\begin{tabular}{|c|c|}
\hline Theme & Comment \\
\hline 1. Child's well-being & $\begin{array}{l}\text { He's a lot happier now } \\
\text { "Completely different child" } \\
\text { Playing and listens a lot more, happier } \\
\text { He's a happier boy } \\
\text { Child anxiety better } \\
\text { He is so much happier in himself } \\
\text { She's happy and chirpy now } \\
\text { More relaxed and calmer } \\
\text { No longer tired and grumpy }\end{array}$ \\
\hline 2. Parent's well-being & $\begin{array}{l}\text { Mum's sleep a lot better now } \\
\text { "I feel on top of the world" } \\
\text { "I'm in control now" } \\
\text { My anxiety is a lot better } \\
\text { Mum's mental health better }\end{array}$ \\
\hline 3. Sibling's well-being & $\begin{array}{l}\text { Second sibling now sleeping through the night as a result of implementing plan } \\
\text { Plays/cuddles with his sister when he wouldn't before }\end{array}$ \\
\hline 4. Family relationships & $\begin{array}{l}\text { Changed our life/relationship } \\
\text { It was having such a negative impact on the whole of our lives - you've saved it all } \\
\text { Relationship with partner a lot better now } \\
\text { "Happier household, having quality time now" } \\
\text { Happy to visit grandparents separate from mum } \\
\text { Gets more time with husband } \\
\text { Spend quality time together } \\
\text { We are so much happier as a family } \\
\text { Spend time with my husband } \\
\text { We have time for us and we go to the gym now! }\end{array}$ \\
\hline 5. Mental and physical well-being & $\begin{array}{l}\text { Physical and mental health better. More energy } \\
\text { No more cold sores } \\
\text { Mum's physical and mental health improved } \\
\text { Mums migraines reduced a little. } \\
\text { His speech has improved } \\
\text { Gets fewer colds }\end{array}$ \\
\hline 6. School and productivity & $\begin{array}{l}\text { Nursery commented they're happy } \\
\text { (School) attendance has gone up to } 96 \% \text { from } 79 \% \text {, surpassed expected level grades } \\
\text { Willing to go to school now } \\
\text { Child school work improved especially maths } \\
\text { Concentration better } \\
\text { Child sitting down and participating more in school now. improved attention span }\end{array}$ \\
\hline 7. Foster placement & "The social worker said she's maybe not the placement for you, but sleep has enabled her to stay. Maybe forever" \\
\hline 8. Driving & Significant impact on mums driving "in the past I had done journeys and not remembered the way I'd gone" \\
\hline
\end{tabular}

